\title{
iCETL
}

International Conference on Research in Education, Teaching and Learning

Paris, France| November 2-4, 2018

\section{Effective Public Health Twitter Messaging in a Diabetes Health Promotion Campaign}

\author{
Hossam Alakhrass \\ Prof. Azeem Majeed, and Dr. Nikolaos Mastellos, \\ Imperial College London, London, UK
}

\begin{abstract}
Local health departments have been challenged to find effective ways of conducting health promotion using social media. Because $40 \%$ of Twitter accounts in the Arab region originate in the Kingdom of Saudi Arabia (KSA), Twitter was chosen as the social media platform for the KSA Ministry of Health's (MoH's) "Do Not Wait for Diabetes" (DNWD) health promotion campaign which began in November 2017. The existing Twitter account for the MoH's National Diabetes Prevention and Control Program (NDPCP) was assigned to a doctoral student who is advised by a team with diabetes expertise that approves tweets before they are sent. Experiments with techniques to increase the number of "impressions" (number of times the tweet is seen) and "engagements" (the number of times an account engages with a tweet) were conducted. Twitter analytics is used to assess these techniques' efficacy. To promote diabetes-related events in particular geographic areas, tweets about these events can mention other local area accounts, such as for schools and local healthcare facilities. The NDPCP account has gained credibility and visibility through high-profile accounts with many followers retweeting the NDPCP tweets. Creating high-quality tweets that meet the information needs of users has also increased both impressions and engagements. Although the account continues to send approximately 2-3 tweets per day, the use of these techniques has increased impressions and engagements by over 100\% monthly. Follow-up studies are planned to see if the DNWD Twitter campaign is associated with an increase in diabetes screenings at $\mathrm{MoH}$ healthcare facilities.
\end{abstract}

